\title{
PRISCILLA GRIFFO
}

Anticorpos anti-DNase I: nova reatividade sorológica na síndrome de Sjögren primária

Dissertação apresentada à Faculdade de Medicina da Universidade de São Paulo para obtenção do título de Mestre em Ciências

Programa de Ciências Médicas Área de concentração: processos imunes e infecciosos

Orientadora: Prof ${ }^{a}$.Drª . Eloisa Silva Dutra de Oliveira Bonfá

São Paulo

2018 


\section{Dados Internacionais de Catalogação na Publicação (CIP)}

Preparada pela Biblioteca da

Faculdade de Medicina da Universidade de São Paulo

Creprodução autorizada pelo autor

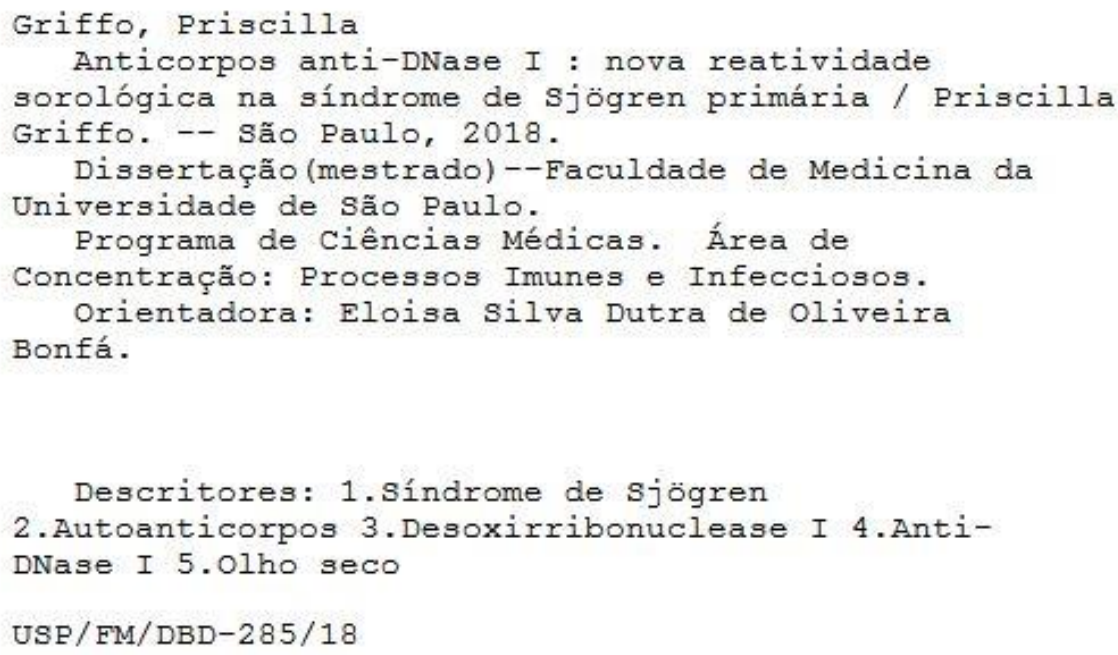


Dedico esse trabalho a todos os profissionais que almejam o bem dos pacientes como fim último a ser alcançado.

E também aos pacientes com doenças autoimunes. Se há um fim nobre na ciência, é fazer descobertas que auxiliem nossos irmãos em dificuldades

Os avanços que buscamos são uma obrigação, enquanto virmos o meio científico como lastreador da evolução civilizatória. E, neste campo, não pode nunca prevalecer a vaidade em detrimento do bem coletivo. 


\section{AGRADECIMENTOS}

Em primeiro plano, agradeço ao Deus do meu coração e da minha compreensão por ser meu guia e fonte de toda minha existência.

A conclusão dessa pesquisa seria impossível sem a colaboração de pessoas e instituições que fizeram parte desse processo. Destas manifesto um agradecimento especial.

A meus pais, pela vida, pelo exemplo de retidão e pelo aprendizado da honestidade como valor inolvidável.

A Lucas Tavares pelo apoio durante o processo.

A minha filha, Maria Laura, por ser um exemplo de luta, perseverança e por me mostrar todos os dias que superar e seguir em frente é o único caminho.

A minha orientadora, Professora Doutora Eloisa Bonfá, por ser um grande exemplo de liderança, pelo suporte no pouco tempo que lhe coube, pelo incentivo e pelas correções. Este trabalho é fruto de uma jornada intensa de aprendizado, sempre sob orientação competente e atenciosa.

A minha co-orientadora Dra. Vilma dos Santos Trindade Viana, por ter me acolhido, pelos ensinamentos importantíssimos para minha evolução acadêmica, paciência e por ser um exemplo de excelente profissional e raro ser humano - capaz de se doar altruisticamente para além das obrigações inerentes as suas funções. 
A minha co-orientadora clínica Dra. Sandra Gofinet Pasoto. Sem seu suporte na área clínica, dado com detalhismo e brilhantismo, este trabalho não teria sido possível.

A Elaine Pires Leon, por toda a ajuda na bancada, pela paciência em me ensinar e acompanhar.

A Cleonice Bueno, Margarete Borges Galhardo Vendramini, Elislaine Fiore Garcia dos Reis, Nicole Andressa Gomes Fontoura, Ana Paula Rossi Gandara, Valéria Caparbo, Ana Paula Velosa, Renata Pisani por todo o apoio nos momentos fáceis e difíceis desta jornada.

Às secretárias Claudia Reis de Oliveira, Marta Janete Pereira e Mayra de Carvalho, por toda paciência e orientação na parte burocrática.

Às colegas de jornada, Gabriela Rezende, Renata Rosa, Luciana Parente Costa, Marilda Guimarães, por todo o apoio acadêmico, pela amizade e força durante todo o processo.

A toda equipe do LIM 17, pela disposição sempre presente em ajudar.

A esta universidade e todo seu corpo docente.

Às equipes multidisciplinares da obstetricia, neonatologia e neurologia, que cuidaram tão bem de mim, da Maria Laura e do meu pai durante o tempo em que ficamos internados no Complexo do HC; sem este auxílio, provavelmente não estaria viva para concluir este trabalho.

Um agradecimento em destaque aos meus primeiros incentivadores na vida acadêmica: Professor Mark Huxham, Dr. Lorna Proudfoot, Dr. Mick Rae, Jacqueline Christoforou, da Edinburgh Napier University, Dr. Achim Schnaufer, Caroline Dewar da Edinburgh University, Professor John Underhill da Heriot Watt 
University, ao Sr. Nelson Fernandes e Marilene Gomes Garritano, que sempre vibrou com cada conquista que obtive tanto aqui quanto em terras estrangeiras.

Finalmente, meu muito obrigado a todos que direta ou indiretamente fizeram parte dessa minha trajetória e me fizeram crescer como profissional e ser humano. 


\section{AGRADECIMENTOS ESPECIAIS}

À CAPES (Coordenação de Aperfeiçoamento de Pessoal de Nível Superior) que aprovou e financiou a execução desse projeto, pela confiança que dedica aos pesquisadores e à pesquisa cientifica 
"Where there is a will there is a way".

(An English proverb) 
Esta dissertação está de acordo com as seguintes normas, em vigor no momento desta publicação:

Referências: adaptado de International Committee of Medical Journals Editors (Vancouver).

Universidade de São Paulo. Faculdade de Medicina. Serviço de Biblioteca e Documentação. Guia de apresentação de dissertações, teses e monografias.

Elaborado por Anneliese Carneiro da Cunha, Maria Julia de A. L. Freddi, Maria F. Crestana, Marinalva de Souza Aragão, Suely Campos Cardoso, Valéria Vilhena. $3^{a}$ ed. São Paulo: Divisão de Biblioteca e Documentações; 2011.

Abreviatura dos títulos dos periódicos de acordo com List of Journals Indexed in Index Medicus. 


\section{SUMÁRIO}

Lista de figuras

Lista de tabelas

Lista de abreviaturas e siglas

Resumo

Abstract

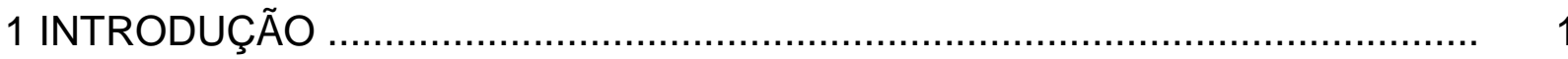

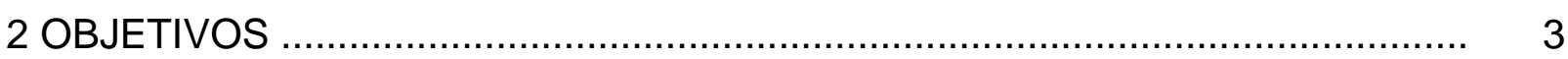

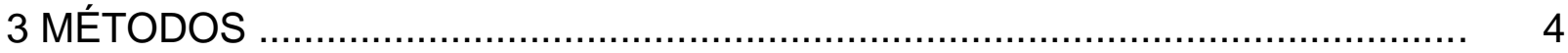

3.1 Grupos de pacientes com SSp, pacientes com AR e controles saudáveis... 4

3.2 Avaliação Clínica ......................................................................... 5

3.3 Determinações laboratoriais.......................................................... 6

3.4 Determinação do anticorpo lgG sérico dirigido à DNase I ........................ 6

3.5 Análise estatística .................................................................... 8

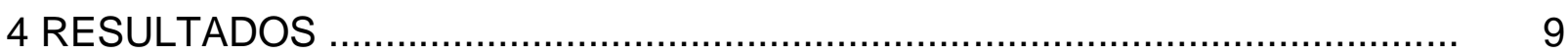

4.1 Características demográficas, clínicas e laboratoriais

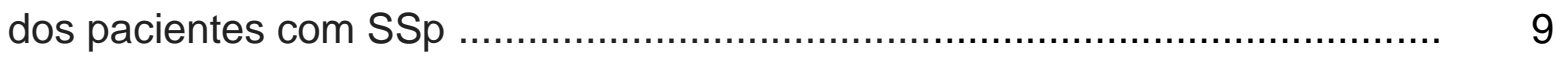

4.2 Comparação dos pacientes com SSp com e sem anticorpo anti-DNase I .............................................................. 10

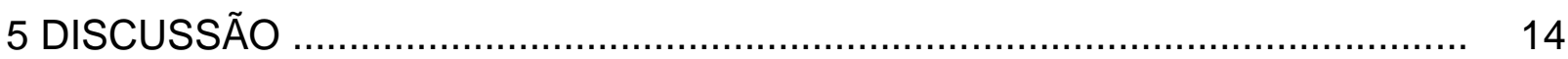

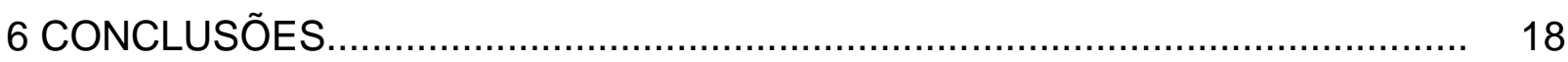

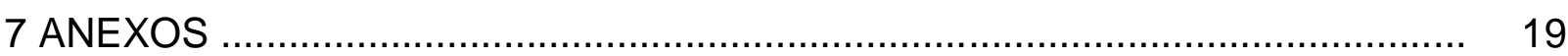

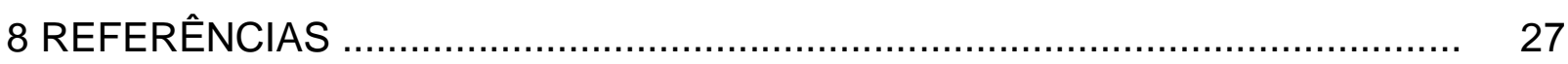

Apêndice 


\section{LISTA DE FIGURAS}

FIGURA 1.Padrão de reatividade dos anticorpos lgG anti-DNase I pancreátca bovina por Immunoblotting. 


\section{LISTA DE TABELAS}

TABELA 1.Características clínicas e demográficas dos pacientes com SSp com e sem anticorpos IgG anti-DNase I

TABELA 2.Autoanticorpos em pacientes com SSp com e sem anticorpos IgG anti-DNase I 


\section{LISTA DE ABREVIATURAS E SIGLAS}

Anti-dsDNA Anticorpo anti-DNA de dupla fita

AR $\quad$ Artrite Reumatoide

BCIP 5-bromo-4-chloro-3-indolyl-phosphate/ 5-bromo-4-cloro-3-indolil-fosfato

BSA Bovine Serum Albumin/ Albumina de soro bovino

DNase I Desoxirribonuclease tipo I

DO Densidade óptica

DP Desvio padrão

ELISA Enzyme-linked immunosorbent assay/ Ensaio imunoenzimático

ESSDAI EULAR Sjögren's Syndrome Disease Activity Index

EULAR European League Against Rheumatism

LES Lúpus eritematoso sistêmico

NBT Nitro blue tetrazolium/ Nitro azul tetrazólio

PBS Phospate buffered saline/ Solução salina tamponada com tampão fosfato

SDS-PAGE Sodium dodecyl sulfate-polyacrylamide gel electrophoresis/

Eletroforese em gel de poliacrilamida sob sódio dodecil sultato

SSp

Síndrome de Sjögren primária

TBS Tris buffered saline/ Solução salina tamponada com tampão Tris 


\section{RESUMO}

Griffo P. Anticorpos Anti-DNase I: nova reatividade sorológica na síndrome de Sjögren primária [dissertação]. São Paulo: Faculdade de Medicina, Universidade de São Paulo; 2018.

INTRODUÇÃO: A síndrome de Sjögren primária (SSp) é uma doença autoimune inflamatória crônica que afeta principalmente as glândulas exócrinas, levando aos sintomas de síndrome sicca. O olho seco é uma das características mais importantes dessa síndrome e um estudo recente relatou redução da atividade da DNase I em lágrimas de pacientes com olho seco de várias etiologias. Portanto, postulamos que pacientes com SSp possam ter anticorpos direcionados à DNase I. MÉTODOS: Avaliamos em um estudo de corte transversal 85 pacientes com SSp (conforme os critérios de classificação do American European Consensus Group Criteria, 2002), 50 pacientes com artrite reumatoide (AR) (American College of Rheumatology Criterial 1987) sem sintomas de síndrome sicca e 88 voluntários saudáveis. A reatividade IgG antiDNase I foi detectada por ELISA utilizando a enzima de pâncreas bovino como antígeno e confirmada por Imunoblotting. RESULTADOS: A idade e sexo foram comparáveis nos três grupos ( $p>0,05)$. A anti-DNase I foi detectada em 43,5\% dos pacientes com SSp, conforme determinado por ELISA. Em contraste, essa reatividade estava ausente em todos os pacientes com AR $(p=0,0001)$. Comparações adicionais dos pacientes com SSp com $(n=37)$ e sem $(n=48)$ antiDNase I revelaram que o primeiro grupo tinha níveis séricos de lgG mais altos $(2293,2 \pm 666,2$ vs. $1483,9 \pm 384,6 \mathrm{mg} / \mathrm{dL}, \mathrm{p}=0,0001)$ e uma frequência maior 
de leucopenia não induzida por drogas $(43 \%$ vs. $19 \%, p=0,02)$. A análise de regressão logística multivariada mostrou que apenas os níveis de IgG foram independentemente associados com 0 anti-DNase I. CONCLUSÃO: Descrevemos uma alta frequência de anticorpos anti-DNase I em pacientes com SSp associados a níveis séricos de IgG mais elevados. A falta dessa reatividade em pacientes com AR sem sintomas de sicca sugere que esse anticorpo pode ser útil no diagnóstico diferencial dessas doenças.

Descritores: Síndrome de Sjögren, autoanticorpos, desoxirribonuclease I, antiDNase I, olho seco. 


\section{ABSTRACT}

Griffo P. Anti-DNase I antibody: new serological reactivity in primary Sjögren's syndrome [dissertation]. São Paulo: "Faculdade de Medicina, Universidade de São Paulo"; 2018.

INTRODUCTION: Primary Sjögren's syndrome (pSS) is a chronic inflammatory autoimmune disease that mainly affects exocrine glands. Dry eye is one of the most important features of this syndrome and a recent study reported reduced DNase I activity in tears of patients with dry eye of various etiologies. We therefore postulated that patients with pSS may have antibodies targeting DNase I. METHODS: We have evaluated in a cross-sectional study 85 pSS patients (American European Consensus Group Criteria/ 2002), 50 rheumatoid arthritis (RA) patients (American College of Rheumatology Criteria/ 1987) without sicca symptoms and 88 healthy volunteers. The IgG anti-DNase I reactivity was detected by ELISA using bovine pancreas enzyme as antigen and confirmed by Immunoblotting. RESULTS: Age/ gender were comparable in the three groups ( $p>0.05$ ). Anti-DNase I was detected in $43.5 \%$ of the pSS patients as determined by ELISA. In contrast, this reactivity was absent in all RA patients $(p=0.0001)$. Further comparison of pSS patients with $(n=37)$ and without $(n=48)$ anti-DNase I revealed that the former group had higher lgG serum levels (2293.2 \pm 666.2 vs. $1483.9 \pm 384.6 \mathrm{mg} / \mathrm{dL}, \mathrm{p}=0.0001)$ and a higher frequency of non-drug induced leukopenia (43\% vs. $19 \%, p=0.02$ ). A multivariate logistic regression analysis identified that only lgG levels were independently associated with anti-DNase I. CONCLUSION: We describe a high frequency of anti-DNase I antibodies in pSS 
patients associated with higher serum IgG levels. The lack of this reactivity in RA patients without sicca symptoms suggests that this antibody may be helpful in the differential diagnosis of these diseases.

Descriptors: Sjögren's syndrome, autoantibodies, deoxyribonuclease I, antiDNase I, dry eye. 


\section{INTRODUÇÃO}

A síndrome de Sjögren primária (SSp) é uma doença autoimune inflamatória crônica caracterizada principalmente pelo envolvimento das glândulas exócrinas, levando aos sintomas da síndrome sicca. Além das glândulas exócrinas, vários outros órgãos também podem ser afetados, causando um amplo espectro de manifestações clínicas. A maioria dos pacientes apresenta sintomas extraglandulares leves, como fadiga, poliartralgia e mialgia difusa, mas outros desenvolvem comprometimentos sistêmicos graves, tais como pneumonite, vasculite, neuropatia periférica, glomerulonefrite, neurite óptica, doença semelhante à esclerose múltipla e até mesmo linfoma (Mariette X., Criswell L.A., 2018). A doença acomete predominantemente mulheres (9:1), com pico de incidência entre 40 e 60 anos de idade (García-Carrasco M. et al., 2002) e sua prevalência na população geral é de aproximadamente $0,1-0,5 \%$ (Bowman S.J. et al., 2004; Sánchez-Guerrero J. et al., 2005).

A etiologia da SSp ainda é desconhecida, mas o envolvimento do sistema autoimune é representado pela produção de um amplo painel de autoanticorpos. Os anticorpos anti-Ro/SSA e anti-La/SSB são os mais comumente encontrados na SSp com uma frequência de até $90 \%$ e $60 \%$, respectivamente (Fayyaz A. et al., 2016) e ambos estão incluidos nos critérios de classificação dessa síndrome (Vitali C. et al., 2002; Shiboski C.H. et al., 2017).

O olho seco é uma manifestação muito importante da SSp e é de grande interesse o recente relato sobre a associação entre a redução da atividade enzimática da desoxirribonuclease I (DNase I) na lágrima dos pacientes com olho 
seco de várias causas, particularmente na doença do enxerto versus hospedeiro (Sonawane S. et al., 2012). A DNase I é uma enzima extracelular capaz de hidrolisar preferencialmente a molécula de DNA de fita dupla para liberar oligonucleotídeos e possui papel importante na eliminaçao de detritos apoptoticos (Samejima K, Earnshaw W.C., 2005; Hakkim A. et al., 2010). Há também evidências de que a DNase I, na forma de colírio utilizado para o tratamento do olho seco, resultou em diminuição da inflamação da superfície ocular, com consequente melhora dos sintomas e sinais de olho seco (Tibrewal S. et al., 2013).

É importante ressaltar que a presença dos anticorpos anti-DNAse I foi relatada nos soros de pacientes com lúpus eritematoso sistêmico (LES) (Yeh T.M. et al., 2003), mas não há dados sobre essa reatividade nos pacientes com SSp, apesar do fato de apresentarem alta frequência de olho seco. 


\section{OBJETIVOS}

O objetivo desse estudo, portanto, foi avaliar a possível presença de anticorpos dirigidos à DNase I nos soros de pacientes com SSp e suas associações clínicas e laboratoriais. Além disso, avaliamos a possível presença dessa reatividade em pacientes com artrite reumatoide (AR) sem queixas de síndrome sicca, a fim de determinar se os anticorpos anti-Dnase I poderiam ser úteis no diagnóstico diferencial dessas condições. 


\section{MÉTODOS}

\subsection{Grupos de pacientes com SSp, pacientes com AR e controles saudáveis}

Avaliamos em um estudo de corte transversal 85 pacientes com SSp, 88 voluntários saudáveis e 50 pacientes com AR.

Oitenta e cinco pacientes consecutivos com SSp de ambos os sexos, com idades entre 18 e 69 anos, que preencheram os critérios internacionais de classificação para a SSp (The American-European Consensus Group Criteria, 2002), (Vitali C. et al., 2002), acompanhados no Ambulatório de Síndrome de Sjögren do Serviço de Reumatologia do Hospital das Clínicas da Faculdade de Medicina da Universidade de São Paulo (HCFMUSP) foram recrutados conforme previamente descrito (Pasoto S.G. et al., 2012; Pasoto S.G. et al., 2013). Os critérios de exclusão foram: 1) outras doenças reumáticas autoimunes associadas, como LES, esclerose sistêmica, polimiosite/ dermatomiosite, AR e doença mista do tecido conjuntivo; 2) presença de sarcoidose, história de radioterapia de cabeça e pescoço, doença do enxerto versus hospedeiro e uso de medicamentos associados à síndrome sicca; 3) presença de anticorpos específicos do LES [anti-DNA de cadeia dupla (anti-dsDNA) e anti-Sm]; e 4) testes sorológicos positivos para hepatites B, C e HIV. O estudo foi aprovado pela Comissão de Ética para Análise de Projetos de Pesquisa (CAPPesq) (50871115.0.0000.0068) e todos os participantes assinaram o termo de consentimento livre e esclarecido.

Dois grupos controle, incluindo oitenta e oito voluntários saudáveis selecionados entre funcionários do hospital e seus familiares e 50 pacientes com AR estabelecida (preenchendo os critérios de classificação do Colégio 
Americano de Reumatologia de 1987) (van der Linden M.P. et al., 2011), com idade e distribuição de gênero comparáveis às do grupo com SSp também foram recrutados. O critério de elegibilidade para os dois grupos controle foi a ausência de queixas da síndrome sicca na história clínica. Todos assinaram o termo de consentimento livre e esclarecido.

\subsection{Avaliação Clínica}

À inclusão, os pacientes com SSp foram avaliados por um mesmo especialista (S.G.P.) (sem o prévio conhecimento dos resultados do teste de antiDNase I) quanto aos envolvimentos glandular e extraglandular da SSp (constitucional, linfadenopatia, cutâneo, articular, respiratório, cardiovascular, renal e do sistema nervoso central e periférico) através de um protocolo clínico padronizado. Esse protocolo incluiu dados de anamnese, exame físico e exames complementares, além de uma extensa revisão do banco de dados de registro eletrônico (estabelecido em 2000 e realizado a intervalos de 1 a 6 meses, incluindo as variáveis relevantes para o presente estudo). A atividade da doença foi avaliada através do European League Against Rheumatism (EULAR) Sjögren's Syndrome Disease Activity Index (ESSDAI), realizado no mesmo dia em que as amostras de soro foram obtidas (Seror R. et al., 2010). Os tratamentos atuais com antimaláricos, glicocorticoides, fármacos imunossupressores (metotrexato, leflunomida, azatioprina, ciclosporina, micofenolato mofetil e ciclofosfamida) e agentes biológicos também foram registrados.

As características demográficas e clínicas dos pacientes com AR foram obtidas através da revisão do banco de dados de registro eletrônico. 
(estabelecido em 2000 e realizado a intervalos de 1 a 6 meses, incluindo as variáveis relevantes para esse estudo).

\subsection{Determinações laboratoriais}

Os anticorpos antinucleares (FAN) e anti-DNA de dupla fita (antidsDNA) foram detectados por imunofluorescência indireta em células HEp-2 e Crithidia luciliae, respectivamente (INOVA Diagnostics Inc., San Diego, EUA) (Esparza R.H. et al., 1985). Os níveis séricos dos anticorpos anti-Ro/SSA, antiLa/SSB, anti-RNP e anti-Sm foram determinados por ELISA de acordo com as instruções do fabricante (INOVA Diagnostics Inc., San Diego, EUA) (Mahler M. et al., 2005). O fator reumatoide IgM foi detectado pelo teste de aglutinação do látex (Goeldner I. et al., 2010).

Os níveis séricos de lgG e dos componentes do complemento (C3 e C4) foram determinados por imunoturbidimetria (Roche Diagnostics, Indianapolis, EUA) e imunodifusão radial (Siemens Healthcare Diagnostics Products $\mathrm{GmbH}$, Marburg, Alemanha), respectivamente.

\subsection{Determinação do anticorpo IgG sérico dirigido à DNase I}

O anticorpo IgG sérico para a DNase I foi determinado por ensaio imunoenzimático (ELISA) padronizado em nosso laboratório. Resumidamente, microplacas de poliestireno de alta ligação foram revestidas com $100 \mu \mathrm{L}$ de 5 $\mu \mathrm{g} / \mathrm{mL}$ de DNase I purificada a partir de pâncreas bovino (Sigma-Aldrich, St Louis, MO, EUA) diluída em tampão fosfato (PBS). As placas foram incubadas 
durante $1 \mathrm{~h}$ sob agitação à temperatura ambiente e, depois, durante a noite a $4^{\circ} \mathrm{C}$. Essa etapa foi seguida de 3 ciclos de lavagem com PBS e o bloqueio dos sítios livres foi realizado pela adição de $200 \mu \mathrm{L}$ de albumina de soro bovino a $5 \%$ (BSA) (Sigma-Aldrich, St Louis, MO, EUA) por 1 h e 30 min a 37ํ․ Após a lavagem, foram adicionados a cada poço $100 \mu \mathrm{L}$ das amostras de soro diluídas 1:100, seguido de incubação de $1: 30$ h a $37^{\circ} \mathrm{C}$. A ligaçao antígeno-anticorpo foi detectada pela adição de anticorpo de cabra conjugado com fosfatase alcalina dirigido à IgG humana (Sigma-Aldrich, St Louis, MO, EUA). Após a lavagem, a reação foi desenvolvida com a adição do cromógeno (sal dissódico do 4-nitrofenil fosfato), interrompida com ácido clorídico e os valores de densidade óptica (DO) determinados em um leitor de microplacas (SpectraMax 190, Molecular Devices, Silicon Valley, CA, EUA ). Os resultados foram expressos como unidades de DO. O valor de corte foi determinado como média \pm 3 desvios padrão (DP) de 88 amostras de soro de voluntários saudáveis incluídos no estudo $(0,19 \pm 0,33)$. Dois controles positivos selecionados entre os pacientes com SSp testados aleatoriamente quanto à reatividade anti-DNase I e com um valor de DO de 1,0 a 405 nm nos primeiros 15-30 min da reação foram incluídos em cada ensaio.

A reatividade anti-DNase I obtida por ELISA foi ainda confirmada pelo ensaio Immunoblot. A DNase I ( $2 \mu \mathrm{g} / \mathrm{slot})$ foi submetida à eletroforese em gel de poliacrilamida com dodecil sulfato de sódio (SDS-PAGE) 14\% e, depois, transferida para uma membrana de nitrocelulose. Após o bloqueio com BSA a $5 \%$ durante $1 \mathrm{~h}$, as tiras foram incubadas com as amostras de soro diluídas a 1:50 durante 1:30 h à temperatura ambiente. Após a lavagem com PBS, as tiras foram incubadas com lgG de cabra anti-lgG humana conjugada com fosfatase. 
A reação foi desenvolvida pela adição de BCIP (5-bromo-4-cloro-3-indolilfosfato) e NBT (nitro azul tetrazólio).

\subsection{Análise estatística}

A análise estatística foi realizada utilizando o programa GraphPad (@2018 GraphPad Software). A comparação entre dois grupos foi realizada pelo teste t de Student ou pelo teste U de Mann-Whitney para variáveis contínuas e teste do qui-quadrado ou teste exato de Fisher para variáveis categóricas, quando aplicáveis. Os resultados foram apresentados como uma proporção ou média \pm desvio padrão (DP). Apenas testes bicaudais foram aplicados. Valores de $p<0,05$ foram considerados estatisticamente significativos. 


\section{RESULTADOS}

\subsection{Características demográficas, clínicas e laboratoriais dos pacientes com SSp}

A média de idade dos pacientes com SSp foi de 47,6 \pm 10,1 anos, com predomínio do sexo feminino (96,5\%). Os pacientes com SSp foram comparáveis aos pacientes com AR [idade média: 48,9 $\pm 9,5$ anos $(p=0,46)$; sexo feminino: $90 \%(p=0,15)$ ] e aos controles saúdaveis [idade média: 46,3 \pm 12,2 anos $(p=0,45)$; sexo feminino: $90,9 \%(p=0,21)]$, respectivamente.

A duração média da doença foi de $8,9 \pm 5,2$ anos. As frequências de manifestações glandulares e extraglandulares da SSp foram: xeroftalmia e/ou xerostomia (99\%), parotidite (66\%), artrite (59\%), vasculite cutânea (22\%), fenômeno de Raynaud (41\%), doença das pequenas vias aéreas (bronquiolite e/ou pneumonite intersticial) (13\%), acidose tubular renal (4\%), comprometimento do sistema nervoso periférico (7\%), do sistema nervoso central (5\%) e linfoma (4\%). De acordo com o ESSDAI, a maioria dos pacientes $(60 \%)$ foi classificada com baixa atividade (ESSDAI $<5)$, doença de atividade moderada (5 SESSDAI $\leq 13)(29 \%)$ e apenas $11 \%$ com alta atividade da doença $(\mathrm{ESSDAI} \geq 14)$.

Os anticorpos antinucleares foram detectados em $93 \%$ dos pacientes com SSp; o anti-Ro/SSA, em 92\%; o anti-La/SSB, em 72\% e o fator reumatoide, em 39\%. Baixos níveis da fração C4 do complemento foram encontrados em 20\% dos pacientes com SSp, enquanto os níveis de C3 estavam dentro da normalidade em todas as amostras testadas. 
Os anticorpos IgG anti-DNase I foram detectados em quase metade dos pacientes com SSp $(43,5 \%)$ conforme determinado pelo teste de ELISA, com uma DO média de 0,70 $\pm 0,11$ ( $\pm \mathrm{DP})$. Em contraste, esta reatividade esteve ausente em todos os controles saudáveis $(p<0,0001)$ e em todos pacientes com AR $(p=0,0001)$. A reatividade anti-DNase I detectada por ELISA foi confirmada por Imunoblotting (Figura 1).

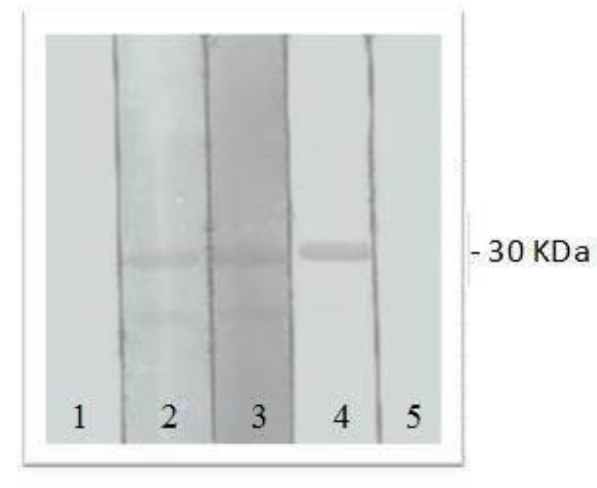

Figura 1. Padrão representativo da reatividade dos anticorpos IgG anti-DNase I pancreática bovina por Immunoblotting. Fitas 1 e 5: soros de dois indivíduos saudáveis; fitas 2, 3 e 4: soros de três pacientes com SSp com anti-DNase I positivo pelo teste de ELISA.

\subsection{Comparação dos pacientes com SSp com e sem anticorpo anti-DNase I}

Comparações entre os pacientes com SSp com $(n=37)$ e sem $(n=48)$ anticorpos IgG anti-DNase I revelaram frequências mais altas de leucopenia não induzida por drogas $(43 \%$ vs. $19 \%, p=0,02)$ e uma tendência à neutropenia $(16 \%$ vs. $4 \%, p=0,07)$. Não foram observadas associações com as características demográficas, duração da doença, manifestações glandulares e extraglandulares e com o escore médio do ESSDAI (Tabela 1). A porcentagem de pacientes com neuropatia periférica foi menor no grupo de pacientes com SSp 
e anti-DNase I positivo do que naqueles sem essa reatividade $(p=0,03)$ (Tabela 1). 
Tabela 1. Características clínicas e demográficas dos pacientes com SSp com e sem anticorpos IgG anti-DNase I.

\begin{tabular}{|c|c|c|c|}
\hline & $\begin{array}{c}\text { Anti-DNase I } \\
\text { positivo } \\
n=37\end{array}$ & $\begin{array}{c}\text { Anti-DNase } \\
\text { negativo } \\
n=48\end{array}$ & $p$ \\
\hline \multicolumn{4}{|l|}{ DADOS DEMOGRÁFICOS } \\
\hline Idade, anos & $47,1 \pm 10,4$ & $48 \pm 9,9$ & 0,68 \\
\hline Sexo feminine & $37(100)$ & $45(94)$ & 0,25 \\
\hline Cor branca & $28(76)$ & $30(63)$ & 0,24 \\
\hline \multicolumn{4}{|l|}{ DADOS CLÍNICOS } \\
\hline Tempo de doença, anos & $9,0 \pm 4,4$ & $8,8 \pm 5,7$ & 0,88 \\
\hline Tempo de seguimento, anos & $5,6 \pm 4,0$ & $5,2 \pm 4,3$ & 0,64 \\
\hline Olho seco & $37(100)$ & $47(98)$ & 1,00 \\
\hline Úlceras de cornea & $1(3)$ & $5(10)$ & 0,23 \\
\hline Sensação de corpo estranho & $34(92)$ & $42(88)$ & 0,73 \\
\hline Uso de lágrimas artificiais & $35(95)$ & $46(96)$ & 1,00 \\
\hline Testes objetivos oculares positivos & $26 / 34(76,5)$ & $37 / 45(82,2)$ & 0,58 \\
\hline Boca seca & $36(97)$ & $48(100)$ & 0,44 \\
\hline Parotidite & $26(70)$ & $30(63)$ & 0,50 \\
\hline Cintilografia de glândulas salivares positiva & $33 / 34(97,1)$ & $41 / 42(97,6)$ & 1,00 \\
\hline Artrite & $25(68)$ & $25(52)$ & 0,19 \\
\hline Artralgia & $33(89)$ & $43(90)$ & 1,00 \\
\hline Vasculite & $8(22)$ & $11(23)$ & 1,00 \\
\hline Envolvimento pulmonary & $3(8)$ & $8(17)$ & 0,33 \\
\hline Acidose tubular renal & $2(5)$ & $1(2)$ & 0,58 \\
\hline Neuropatia periférica & 0 & $6(13)$ & 0,03 \\
\hline Sistema nervoso central & $1(3)$ & $3(6)$ & 0,63 \\
\hline Anemia* & $16(43)$ & $16(33)$ & 0,38 \\
\hline Leucopenia* & $16(43)$ & $9(19)$ & 0,02 \\
\hline Neutropenia* & $6(16)$ & $2(4)$ & 0,07 \\
\hline Linfopenia* & $10(27)$ & $15(31)$ & 0,81 \\
\hline Trombocitopenia* & $3(8)$ & $2(4)$ & 0,65 \\
\hline \multicolumn{4}{|l|}{ ESSDAI } \\
\hline Média \pm DP & $6,1 \pm 5,5$ & $4,7 \pm 6,6$ & 0,28 \\
\hline ESSDAI $\geq 13$ & $4(11)$ & $5(10)$ & 1,00 \\
\hline \multicolumn{4}{|l|}{ COMORBIDADES } \\
\hline Linfoma** & $0(0)$ & $3(6)$ & 0,25 \\
\hline Diabetes & $2(5)$ & $2(4)$ & 1,00 \\
\hline Hipotireoidismo & $16(43)$ & $15(31)$ & 0,27 \\
\hline Hipertireoidismo & $1(3)$ & $2(4)$ & 1,00 \\
\hline \multicolumn{4}{|l|}{ TRATAMENTO ATUAL } \\
\hline Prednisona & $16(43)$ & $19(40)$ & 0,83 \\
\hline Dose diária, mg/dia & $4,2 \pm 7,2$ & $5,0 \pm 11,3$ & 0,46 \\
\hline Antimaláricos & $19(51)$ & $18(38)$ & 0,27 \\
\hline Imunossupressores & $14(38)$ & $16(33)$ & 0,82 \\
\hline
\end{tabular}


Os pacientes com anticorpos anti-DNase I apresentaram níveis séricos de IgG significativamente mais elevados comparativamente àqueles sem essa reatividade $(2293,2 \pm 666,2$ vs. $1483,9 \pm 384,6 \mathrm{mg} / \mathrm{dL}, \mathrm{p}=0,0001)$, com frequências comparáveis de FAN, anti-Ro/SSA e fator reumatoide $(p>0,05)$ (Tabela 2).

A análise de regressão logística múltipla mostrou que, em contraste com leucopenia $(p=0,206)$ e neuropatia periférica $(p=0,999)$, apenas os níveis séricos de lgG permaneceram independentemente associados à presença do anticorpo anti-DNase I $(p<0,001)$.

Tabela 2. Autoanticorpos em pacientes com SSp com e sem anticorpos IgG antiDNase I.

\begin{tabular}{lccc}
\hline & $\begin{array}{c}\text { Anti-DNase I } \\
\text { positivo } \\
\mathrm{n}=\mathbf{3 7}\end{array}$ & $\begin{array}{c}\text { Anti-DNase I } \\
\text { negativo } \\
\mathrm{n}=\mathbf{4 8}\end{array}$ & $\boldsymbol{p}$ \\
\hline AUTOANTICORPOS & & & \\
Anti-Ro/SSA & $36(97)$ & $42(88)$ & 0,13 \\
Anti-La/SSB & $31(84)$ & $30(63)$ & 0,051 \\
Fator reumatoide & $17(46)$ & $16(33)$ & 0,30 \\
ANA & $37(100)$ & $42 / 47(89)$ & 0,06 \\
IgG, mg/DI & $2293,2 \pm 666,2$ & $1483,9 \pm 384,6$ & 0,0001 \\
\hline
\end{tabular}




\section{DISCUSSÃO}

O presente estudo é o primeiro na literatura que demonstrou a presença de autoanticorpos específicos para a enzima DNase I nos soros de pacientes com SSp.

A seleçao rigorosa dos pacientes utilizando não apenas os critérios de classificação da SSp do American-European Consensus Group de 2002 (Vitali C. et al., 2002), como também a exclusão daqueles com anticorpos especificos do LES, permitiu um diagnostico mais preciso da SSp. Na verdade, o LES pode coexistir com a SS (Alani H. et al., 2018) e existem várias outras doenças que mimetizam a SSp (Ramos-Casals M. et al., 2007). Além disso, a inclusão de um grupo controle de pacientes com AR sem sintomas de síndrome sicca foi essencial para discriminar essas doenças. De fato, a SS foi relatada em aproximadamente 19,5\% dos pacientes com AR (Alani H. et al., 2018). Além disso, a idade média comparável dos pacientes com SSp, pacientes com AR e indivíduos saudáveis foi relevante, uma vez que mudanças relacionadas à idade podem levar a uma função reduzida das glândulas exócrinas e sintomas de síndrome sicca (Bouma H.R. et al., 2015). Por outro lado, a inclusão de pacientes com AR unicamente com base na ausência de queixas de síndrome sicca e sem testes objetivos é uma limitação e pode ter subestimado a frequência de SS secundária nos pacientes com AR avaliados no presente estudo.

A alta frequência do anticorpo anti-DNase I observada nos pacientes com SSp contrasta com a sua completa ausência no grupo com AR sem sintomas de síndrome sicca. Assim, esse anticorpo pode ser potencialmente útil 
para o diagnóstico diferencial dessas doenças. Nesse aspecto, tal achado tornase relevante considerando-se um estudo recente envolvendo mais de mil pacientes com SS, no qual foi relatado que aproximadamente metade deles tinham diagnóstico prévio de AR, LES ou mesmo esclerose sistêmica (Rasmussen A. et al., 2016).

No entanto, o anticorpo anti-DNase I não é um marcador sorológico específico para a SSp, uma vez que foi previamente detectado em $62 \%$ dos soros de pacientes com LES pelo método de ELISA usando placas revestidas com DNase bovina (Yeh et al., 2003). Esses autores, entretanto, utilizaram um ponto de corte (2 DP) menor em comparação com 3 DP usado no presente estudo e, portanto, sua frequência relatada dessa especificidade de anticorpo pode ser maior nos controles de saudáveis e nos pacientes com LES (Yeh et al., 2003).

Nossos dados demonstram também que os pacientes com SSp com anticorpos anti-DNase I positivos apresentam níveis séricos de lgG total mais altos em comparação àqueles com anticorpos anti-DNase I negativos, provavelmente representando um subgrupo de pacientes com SSp com resposta imune humoral mais exacerbada. Em consonância com esse achado, os pacientes com anti-DNase I positivo tiveram maior frequência de leucopenia não induzida por drogas e tendência a maior taxa de neutropenia, que são dois parâmetros do domínio hematológico do escore ESSDAI (Seror R. et al., 2010). A falta de associação desse anticorpo com outras manifestações glandulares e sistêmicas da doença pode sugerir que essa reatividade pode ter como alvo particular as células brancas do sangue, como também observado com os anticorpos dirigidos aos receptores muscarínicos do tipo 3 na SSp (He J. et al., 
2011). No entanto, a análise de regressão logística multivariada mostrou que apenas os níveis de IgG foram independentemente associados com o antiDNase I e futuros estudos são necessários para confirmar a importância desse anticorpo para as anormalidades hematológicas na SSp.

A pequena representação de pacientes com SSp com alta atividade da doença no presente estudo impede uma conclusão definitiva sobre a associação com o escore ESSDAI.

Além disso, o achado quase universal da manifestação de olho seco nos pacientes com SSp avaliados nesse estudo dificultou a análise de uma possível associação dos anticorpos anti-DNase I com as queixas de síndrome sicca.

Com relação aos autoanticorpos, não houve associação do anticorpo anti-DNase I e a presença concomitante de ANA, anti-Ro/SSA ou fator reumatoide. A frequência geral observada de $72 \%$ de anti-La/SSB nos pacientes com SSp e ainda mais alta naqueles positivos para anti-DNase I (84\%) contrasta com os dados da literatura (30-60\%) (Fayyaz A. et al., 2016). Esse achado pode ser explicado em parte pelos critérios de inclusão rigorosos e pelo fato dos pacientes incluídos nesse estudo serem provenientes de um centro de referência terciário.

Curiosamente, um estudo recente mostrou que os soros dos pacientes com SSp comparados a indivíduos saudáveis apresentaram a atividade da DNase I reduzida, a qual se correlaciou inversamente com os níveis séricos de nucleossomos e DNA extracelular derivado de processos apoptóticos (Fragoulis G.E. et al., 2015). A causa da atividade enzimática diminuída da DNase I na SSp ainda é desconhecida, mas o envolvimento de potenciais moléculas inibidoras 
presentes no soro desses pacientes tem sido considerado (Fragoulis G.E. et al., 2015). Nesse contexto, é possível que os anticorpos dirigido à DNase I, que se apresentam em alta frequência nos soros de pacientes com SSp como aqui descrito, possam desempenhar um papel importante na alteração da atividade biológica dessa enzima. Essa hipótese é corroborada por estudos anteriores demonstrando que preparações purificadas de anticorpos IgG anti-DNase I isolados de soros de pacientes com LES tem a capacidade de inibir in vitro a atividade enzimática da DNase I (Yeh T.M. et al., 2003). No entanto, essa avaliação laboratorial não foi realizada nos pacientes com SSp. A esse respeito, um estudo recente mostrou que a inibição da atividade da DNase I nos soros de pacientes com LES pode ser devida à ação do anti-DNase I, da actina (um importante inibidor da DNase I) e de outros fatores ainda não identificados (Trofimenko A.S. et al., 2016). 


\section{CONCLUSÕES}

Finalmente, nós demonstramos pela primeira vez que o anticorpo antiDNase I é altamente frequente em pacientes com SSp e está associado a níveis séricos elevados de lgG.

A completa ausência dessa reatividade na AR sem sintomas de síndrome sicca sugere que essa especificidade de anticorpo pode ser útil no diagnóstico diferencial dessas duas condições. Futuros estudos longitudinais de grande porte, incluindo pacientes com SSp e outras doenças autoimunes, são necessários para corroborar nossos achados. 


\section{ANEXOS}

Anexo A. Aprovação do Comitê de Ética Local e Pesquisa Humana da Universidade de São Paulo

HOSPITAL DAS CLINICAS

DA

FACULDADE DE MEDICINA DA UNIVERSIDADE DE SÃO PAULO

DIRETORIA CLINICA

COMISSÄO DE ÉTICA PARA ANÁLISE DE PROJETOS DE PESQUISA -

CAPPesq

CADASTRO DE PROTOCOLO DE PESQUISA

\begin{tabular}{|c|c|}
\hline \multicolumn{2}{|c|}{ Registro (uso reserrado à Secrelaria da CAPPesq) } \\
\hline $\begin{array}{l}\mathrm{N}^{\circ} \text { do Protocolo: } \\
\text { Instituto: } \mathrm{ICHC}\end{array}$ & Tipo: Humanos \\
\hline Registro on-line $n^{0:} 13057$ & Data de Entrada: 23/10/2015 \\
\hline
\end{tabular}

Este projeto envolve:

Médicos ou Funcionários $\mathrm{HC}$ (como sujeitos de pesquisa) ............Sim


1. Título do Protocolo de Pesquisa

ANTICORPOS SÉRICOS DIRIGIDOS A DNase I NA SÍNDROME DE SJÖGREN PRIMÁRIA

2. Palavras-chaves que caracterizam o assunto da Pesquisa

anti-DNase Isindrome de Sjögren

\section{Resumo do Protocolo de Pesquisa:}

Introdução: A síndrome de Sjögren primária (SSp) é uma doença sistêmica inflamatória crônica que acomete principalmente as glândulas lacrimais e salivares, levando à "sindrome sicca". A SSp tem provável etiologia autoimune, destacando-se a produção de vários autoanticorpos, tais como os fatores antinucleares (FAN), anti-Ro/SS-A, anti-La/SS-B, fator reumatoide (FR) e crioglobulinas. A SS pode também se associar a outras doenças autoimunes, como artrite reumatoide (AR) e lúpus eritematoso sistêmico (LES), sendo classificada como SS secundária (SSs). Nessas doenças, se postula que a ação de agentes ambientais em hospedeiros geneticamente predispostos possa desencadear os processos autoimunes. Nesse aspecto, foi demonstrada a mutação em genes envolvidos na expressão e atividade da endonuclease desoxirribonuclease I (DNAse I), que atua na hidrólise da molécula do DNA, em pacientes com LES e SS. Esta mutação associou-se à diminuição da atividade da DNase I no soro dos pacientes e ao aumento do título de anticorpos anti-nucleossomais e anti-dsDNA, que são marcadores sorológicos do LES. A deficiência da atividade da DNAse I pode comprometer o clearance de debris celulares resultantes do processo de apoptose com persistência de DNA extracelular e seu envolvimento na patogênese do LES. Há também evidências que anticorpos 
com especificidade para DNase I com capacidade de inibir a sua atividade enzimática podem ser detectados no soro de pacientes com LES.

Recentemente, foi descrita a redução da atividade da DNase I na lágrima em pacientes com xeroftalmia e esse achado associou-se ao acúmulo de DNA extracelular e infiltrado inflamatório neutrofílico na superfície ocular. Posteriormente, foi mostrado que o uso da DNase I em forma de colírio em pacientes com olho seco resultou na diminuição da inflamação da superfície ocular, levando a uma melhora do olho seco.Objetivos: O presente estudo tem como objetivo avaliar a possível presença de anticorpos dirigidos à DNase I no soro de pacientes com SSp e suas possíveis associações clínicas e laboratoriais.Pacientes e Métodos: Serão analisadas amostras de soro de 100 pacientes com SSp (conforme os critérios do consenso americano-europeu de 2002), 100 pacientes com outras doenças reumáticas (AR e LES) com e sem SS secundária. Participarão também 100 controles saudáveis pareados para sexo e idade, sem queixas de síndrome de sicca. O teste imunoenzimático (ELISA) será padronizado em nosso laboratório empregando a enzima bovina purificada como antígeno. A especificidade da reatividade por ELISA será confirmada posteriormente por Western Blot.

\section{Pesquisador Responsável:}

Professora Doutora Eloisa Dutra de Oliveira Bonfá

http://lattes.cnpq.br/1810614032141202

Graduação: Medicina

Vínculo: FMUSP

\section{Pesquisador Executante:}

Priscilla Griffo

http://lattes.cnpq.br/8048772060098230 
6. Possui co-autores?

Sim

Nome dos co-autores: Dra. Vilma dos Santos Trindade Viana, Dra. Sandra Gofinet Pasoto, Dra. Luciana Parente Costa Seguro, Elaine Pires Leon, Cleonice Bueno

7. Onde a Pesquisa será realizada?

Departamento: Clínica Médica

Disciplina: Reumatologia

LIM: LIM/17 - Lab Investigação em Reumatologia

8. Existe entidade externa envolvida?

Não

9. Possui participação Estrangeira

Não

10. O projeto é multicêntrico

Não

11. Outros serviços/ divisões do HCFMUSP envolvidos na pesquisa

Não

12. Finalidade acadêmica da pesquisa e classificação

Mestrado

Outros:

Orientador: Profa. Dra. Eloisa Bonfa

13. Investigação

Retrospectiva e Prospectiva (Ambos)

14. Materiais e métodos

Laboratorial

Entrevistas e questionários

Prontuários de pacientes 
15. Gênero, classificação da Pesquisa

Clínica

16. Áreas temáticas previstas na Res. $466 / 12$

Nenhuma das alternativas

17. Patrocínio

CAPES

18. Valor do financiamento

$10.000,00$

19. Cronograma de execução da pesquisa

Prazo: 24 meses

20. Assinaturas

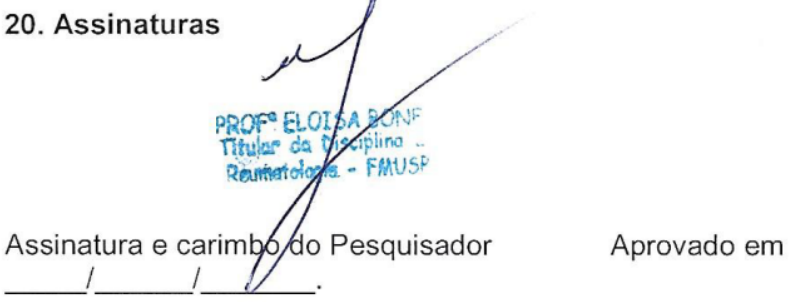

Assinatura e carimbo da Chefia

com data de aprovação

pelo Conselho do Departamento

Aprovado em
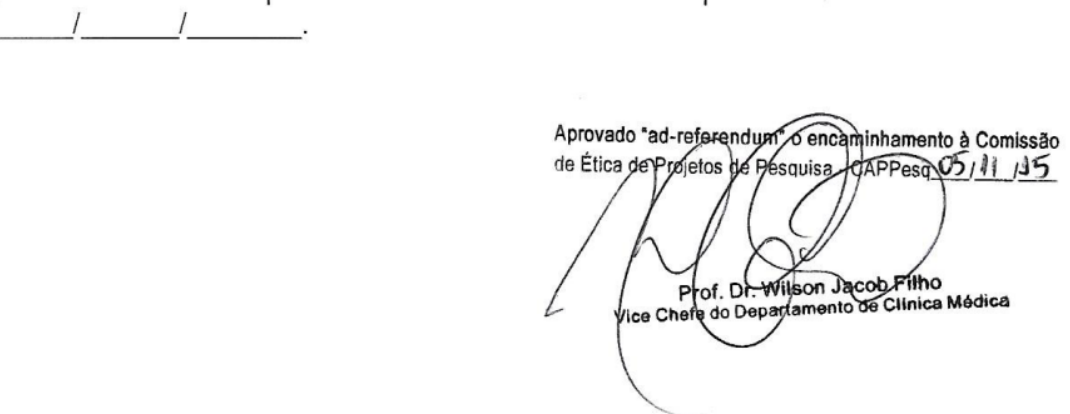


\section{Hospital das Clínicas da FMUSP}

Comissão de Ética para Análise de Projetos de Pesquisa - CAPPesq

Situação do Parecer: Aprovado.

Necessita Apreciação da CONEP: Não.

Considerações Finais a critério do CEP: Em conformidade com a Resolução CNS n 466/12 cabe ao pesquisador: a) desenvolver o projeto conforme delineado; b) elaborar e apresentar relatórios parciais e final; c)apresentar dados solicitados pelo CEP, a qualquer momento; d) manter em arquivo sob sua guarda, por 5 anos da pesquisa, contendo fichas individuais $\mathrm{e}$ todos os demais documentos recomendados pelo CEP; e) encaminhar os resultados para publicação, com os devidos créditos aos pesquisadores associados e ao pessoal técnico participante do projeto; f) justificar perante o CEP interrupção do projeto ou a não publicação dos resultados.

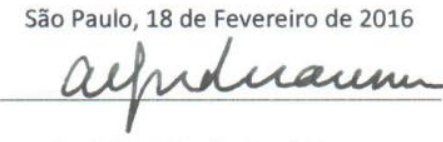

Prof. Dr. Alfredo José Mansur Coordenador

Comissāo de Ética para Análise

de Projetos de Pesquisa - CAPPesq

Rua Dr. Ovídio Pires de Campos, 225 - Prédio da Administração - 5 andar CEP 05403-010 - São Paulo - SP.

5511 2661-7585 - 5511 2661-6442 ramais: 16, 17, 18 | marcia.carvalho@hc.fm.usp.br 


\section{Hospital das Clínicas da FMUSP}

Comissão de Ética para Análise de Projetos de Pesquisa - CAPPesq

\section{PROJETO DE PESQUISA}

Título: ANTICORPOS SÉRICOS DIRIGIDOS A DNASE I NA SÍNDROME DE SJÖGREN PRIMÁRIA Pesquisador Responsável: Professora Doutora Eloisa Dutra Versão: 2 de Oliveira Bonfá

Pesquisador Executante: Priscilla Griffo

CAAE: 50871115.0 .0000 .0068

Coautores: Dra. Vilma dos Santos Trindade Viana, Dra. Sandra Gofinet Pasoto, Dra. Luciana

Parente Costa Seguro, Elaine Pires Leon, Cleonice Bueno

Finalidade Acadêmica: Mestrado

Orientador: Profa. Dra. Eloisa Bonfá

Instituição: HCFMUSP

Departamento: CLIINICA MÉDICA

\section{PARECER CONSUBSTANCIADO DO CEP}

Registro on-line: 13057

Número do Parecer: 1.412 .486

Data da Relatoria: $17 / 02 / 16$

Apresentação do Projeto: Este é um projeto voltado para a síndrome de Sjogren primária.

Objetivo da Pesquisa: Visa testar a presença de anticorpos anti DNAse I no soro, para fins diagnósticos. Para tanto serão analisadas amostras de soro de Sjogren, de outras doenças reumatológicas, e de voluntários sadios.

Avaliação dos Riscos e Benefícios: Não há riscos (estudo retrospectivo).

Comentários e Considerações sobre a Pesquisa: 0 projeto foi corretamente elaborado e é condizente com a literatura científica.

Considerações sobre os Termos de apresentação obrigatória: Não há necessidade de TCLE (material estocado em biorrepositório).

Recomendações: Não há.

Conclusões ou Pendências e Lista de Inadequações: Não há pendências.

Rua Dr. Ovídio Pires de Campos, 225 - Prédio da Administração - 5a andar CEP 05403-010 - São Paulo - SP.

5511 2661-7585 - 5511 2661-6442 ramais: 16, 17, 18 | marcia.carvalho@hc.fm.usp.br 
Plotoformo MINISTÉRIO DA SAÚDE - Conselho Nacional de Saúde - Comissão Nacional de Ética em Pesquisa - CONEP grasi FOLHA DE ROSTO PARA PESQUISA ENVOLVENDO SERES HUMANOS

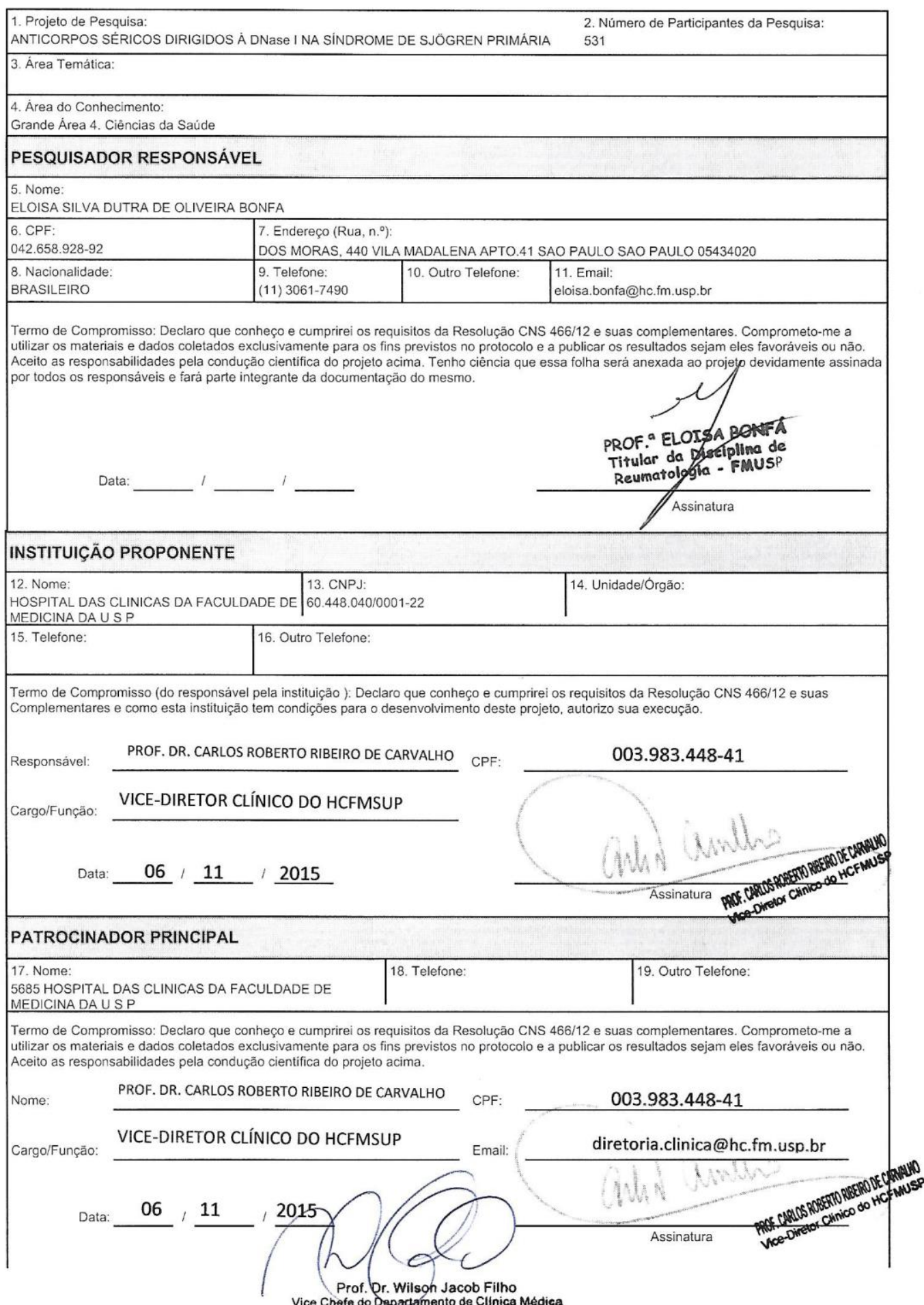




\section{REFERÊNCIAS}

- Alani H, Henty JR, Thompson NL, Jury E, Ciurtin C. Systematic review and meta-analysis of the epidemiology of polyautoimmunity in Sjögren's syndrome (secondary Sjögren's syndrome) focusing on autoimmune rheumatic diseases. Scand J Rheumatol. 2018; 47: 141-154.

- Bouma HR, Bootsma H, van Nimwegen JF, et al. Aging and immunopathology in primary Sjögren's syndrome. Curr Aging Sci. 2015; 8: 202-213.

- Bowman SJ, Ibrahim GH, Holmes G, Hamburguer J, Ainsworth J. Estimating the prevalence among Caucasian women of primary Sjögren's syndrome in two general practices in Birmingham, UK. Scand J Rheumatol. 2004; 33: 3943.

- Esparza RH, Swaak T, Aarden L, Smeenk R. Complement fixing antibodies to dsDNA detected by the immunofluorescence technique on Crithidia luciliae. A critical appraisal. J Rheumatol. 1985; 12: 1109-1117.

- Fayyaz A, Kurien BT, Scofield RH. Autoantibodies in Sjögren's Syndrome. Rheum Dis Clin North Am. 2016; 42: 419-434.

- Fragoulis GE, Vakrakou AG, Papadopoulou A, et al. Impaired degradation and aberrant phagocytosis of necrotic cell debris in the peripheral blood of patients with primary Sjögren's syndrome. J Autoimmun. 2015; 56: 12-22.

- García-Carrasco M, Ramos-Casals M, Rosas J, et al. Primary Sjögren syndrome: clinical and immunologic disease patterns in a cohort of 400 patients. Medicine 2002; 81: 270-280. 
- Goeldner I, Skare TL, de Messias Reason IT, Nisihara RM, Silva MB, Utiyama SR. Anti-cyclic citrullinated peptide antibodies and rheumatoid factor in rheumatoid arthritis patients and relatives from Brazil. Rheumatology (Oxford) 2010; 49: 1590-1593.

- Hakkim A, Fürnrohrb BG, Amannd K, et al. Impairment of neutrophil extracellular trap degradation is associated with lupus nephritis. Proc Natl Acad Sci USA. 2010; 107: 9813-9818.

- He J, Guo JP, Ding Y, et al. Diagnostic significance of measuring antibodies to cyclic type 3 muscarinic acetylcholine receptor peptides in primary Sjogren's syndrome. Rheumatology (Oxford) 2011; 50: 879-884.

- Mahler M, Stinton LM, Fritzler MJ. Improved serological differentiation between systemic lupus erythematosus and mixed connective tissue disease by use of a SmD3 peptide-based immunoassay. Clin Diagn Lab Immunol. 2005; 12: 107-113.

- Mariette X, Criswell LA. Primary Sjögren's syndrome. N Engl J Med. 2018; 378: 931-939.

- Pasoto SG, Chakkour HP, Natalino RR, et al. Lupus anticoagulant: a marker for stroke and venous thrombosis in primary Sjögren's syndrome. Clin Rheumatol. 2012; 31: 1331-1338.

- Pasoto SG, Natalino RR, Chakkour HP, et al. EBV reactivation serological profile in primary Sjögren's syndrome: an underlying trigger of active articular involvement? Rheumatol Int. 2013; 33: 1149-1157.

- Ramos-Casals M, Brito-Zerón P, Font J. Lessons from diseases mimicking Sjögren's syndrome. Clin Rev Allergy Immunol. 2007; 32: 275-283. 
- Rasmussen A, Radfar L, Lewis D, Grundahl K, et al. Previous diagnosis of Sjögren's Syndrome as rheumatoid arthritis or systemic lupus erythematosus. Rheumatology (Oxford) 2016; 55: 1195-1201.

- Samejima K, Earnshaw WC. Trashing the genome: the role of nucleases during apoptosis. Nat Rev Mol Cell Biol. 2005; 6: 677-688.

- Sánchez-Guerrero J, Pérez-Dosal MR, Cárdenas-Velázquez F, et al. Prevalence of Sjögren's syndrome in ambulatory patients according to the American-European Consensus Group criteria. Rheumatology 2005; 44: 235240.

- Seror R, Ravaud P, Bowman SJ, et al., EULAR Sjögren's Task Force. EULAR Sjogren's syndrome disease activity index: development of a consensus systemic disease activity index for primary Sjogren's syndrome. Ann Rheum Dis. 2010; 69, 1103-1109.

- Shiboski CH, Shiboski SC, Seror R, et al. 2016 American College of Rheumatology/European League Against Rheumatism classification criteria for primary Sjögren's syndrome: a consensus and data-driven methodology involving three international patient cohorts. Ann Rheum Dis. 2017; 76: 9-16.

- Sonawane S, Khanolkar V, Namavari A, et al. Ocular surface extracellular DNA and nuclease activity imbalance: a new paradigm for inflammation in dry eye disease. Invest Ophthalmol Vis Sci. 2012; 13: 8253-8263.

- Tibrewal S, Sarkar J, Jassim SH, et al. Fluid extracellular DNA: diagnostic and therapeutic implications in dry eye disease. Invest Ophthalmol Vis Sci. 2013; 54: 8051-8061. 
- Trofimenko AS, Gontar IP, Zborovsky AB, Paramonova OV. Anti-DNase I antibodies in systemic lupus erythematosus: diagnostic value and share in the enzyme inhibition. Rheumatol Int. 2016; 36: 521-529.

- van der Linden MP, Knevel R, Huizinga TW, van der Helm-van, Mil AH. Classification of rheumatoid arthritis: comparison of the 1987 American College of Rheumatology criteria and the 2010 American College of Rheumatology/European League Against Rheumatism criteria. Arthritis Rheum. 2011; 63: 37-42.

- Vitali C, Bombardieri S, Jonsson R, et al. Classification criteria for Sjögren's syndrome: a revised version of the European criteria proposed by the American-European Consensus Group. Ann Rheum Dis. 2002; 61: 554-558.

- Yeh TM, Chang HC, Liang CC, Wu JJ, Liu MF. Deoxyribonuclease-inhibitory antibodies in systemic lupus erythematosus. J Biomed Sci. 2003; 10: 544551. 


\section{Apêndice}

\section{Aceite da Revista Journal Clinical of Rheumatology}

O artigo científico foi submetido ao Journal Clinical of Rheumatology e aceito em 30/07/2018, conforme notificação em anexo.

Jul 302018 12:19:25:378PM

RE: JCR-18-410R2, entitled "ANTI-DNase I ANTIBODY: A NEW SEROLOGICAL REACTIVITY IN PRIMARY SJÖGREN'S SYNDROME"

Dear Prof. Bonfa,

I am pleased to inform you that your manuscript has been accepted for publication in the JCR: Journal of Clinical Rheumatology.

Thank you for contributing your article to the JCR: Journal of Clinical Rheumatology.

The JCR is a peer-reviewed semiquarterly journal listed on Medline and dedicated to the study of rheumatologic and musculoskeletal diseases. Each issue contains original articles, case reports, reviews, expert commentary, letters to the editors and more, providing useful clinical information to practicing clinicians, like you.

We encourage you to subscribe yourself and check if your group's/hospital's/institution's library subscribes to the JCR. Go to our website www.jclinrheum.com home page and click on "New Subscription" and subscribe to find the latest practical reports on diagnosis and patient management issues.

You will be receiving proofs approximately 2 months prior to the publication date from the publisher with directions on returning them. Please check proofs very carefully, as copy editors have been known to misinterpret words or phrases. Please return the proofs within 48 hours so as not to delay publication.

If you indicated in the revision stage that you would like your submission, if accepted, to be made open access, please go directly to step 2. If you have not yet 
indicated that you would like your accepted article to be open access, please follow the steps below to complete the process:

1. Notify the journal office via email that you would like this article to be available open access. Please send your Email to monahanjcr@gmail.com. Please include your article title and manuscript number.

2. A License to Publish (LTP) form must be completed for your submission to be made available open access. Please download the form

from http://links.Iww.com/LWW-ES/A49, sign it, and Email the completed form to the journal office.

\section{Within $\mathbf{4 8}$ hours of receiving this e-mail: Go}

to http://wolterskluwer.qconnect.com to pay for open access. If you have not previously used this site to place an order, you will need to register for an account (your login will be different from your Editorial Manager login). When placing your order, you will be asked for the following information. Please enter exactly as shown:

a. Article Title - ANTI-DNase I ANTIBODY: A NEW SEROLOGICAL REACTIVITY IN PRIMARY SJÖGREN'S SYNDROME

b. Manuscript Number - JCR-18-410R2

Thank you for allowing us to publish your interesting and important work in the JCR.

https://jcr.editorialmanager.com/

Your username is: EBONFA

click here to reset your password

With Kind Regards,

Dr. Graciela S. Alarcón

Editor-in-Chief

JCR: Journal of Clinical Rheumatology

If you would like your personal information to be removed from the database, please contact the publication office. 